\title{
ATTITUDES TO WORKPLACE DIVERSITY - MAIN FINDINGS IN A GLOBAL CONTEXT
}

\author{
Radu-Dan IRINI ${ }^{a *}$, Anca BORZA ${ }^{b}$ \\ ${ }^{a},{ }^{b}$ Babeș-Bolyai University, Romania
}

\begin{abstract}
One of the focal points of research in the diversity management discourse has been analyzing how the managers and other decision-makers perceived diversity while less focus has been placed on the employee's attitudes toward workplace diversity. In order to develop the present analysis, a systematic review was chosen to investigate previous literature on diversity management, workplace diversity, and employee attitudes toward diversity. The first objective of our paper is to identify if there is an alignment between the main findings of the studies that focused on measuring the attitudes to workplace diversity, determining the relation between context and diverse attitudes. The second objective is to analyze the preferred methodologies used by researchers to gather empirical evidence as the subject of measuring attitudes to workforce diversity has been fairly understudied in the diversity management discourse. The findings from 14 national contexts suggest either neutral or positive attitudes to workplace diversity, with a high influence of context and modest generalizability of the results.
\end{abstract}

KEYWORDS: context, diversity, employee attitudes, workforce diversity.

\section{INTRODUCTION}

Nowadays workplace diversity is the norm, being an expected element of the organizational culture and part of the business strategy in most countries. Organizations must be ready to employ diverse individuals, retain talent, and act according to the attitudes related to diversity.

Narrowly defined diversity could be described as all the demographic differences of a given population. Today, diversity concept takes into account a multitude of dimensions, including intangible aspects such as professional experience, religion, and lifestyle (Mohr \& Shoobridge, 2011). Zapata-Barrero and Van Ewijk (2011) suggest that it is the duty of scholars and researchers to create their own version of diversity definition, based on specific implications, scope and context of the study. In our study, we define workplace diversity as the complete range of visible and invisible characteristics that differentiate coworkers.

Diversity in the organizational context could be best understood when acknowledging the important concept of diversity management, considered one of the main keys to growth in today's business environment (Yang \& Konrad, 2011).

Diversity management could be described as the exploration of all diversity dimensions inside the workplace in a safe and fostering environment. With diversity management the organizational goals surpass the simple tolerance of the different other moving to embrace all diversity dimensions found in each individual (Patrick \& Kumar, 2012).

As diverse individuals bring unique perspectives to the organizational context, diversity inside the workplace represent a double-edged sword (Köllen, 2019). Workforce diversity can lead to

\footnotetext{
* Corresponding author. E-mail address: radu.irini@econ.ubbcluj.ro
} 
increased team/organizational performance, enhanced innovation, and creativity (McGuire \& Bagher, 2010), however, the presence of diversity inside organizations could also lead to an increase of conflicts, poor communication, and reduced coordination (Chow, 2018). Besides the legal and ethical reasoning, nowadays more organizations adopt diversity management strategies for the business advantages brought by workplace diversity (Alserhan et al., 2010). Patrick and Kumar (2012) propose that organizations characterized by a diverse set of employees have more expertise to act in an increasingly global market. such organizations are more prepared legally, economically, socially and can offer their services more effectively for their customers and partners. Sü $\beta$ and Kleiner (2007) consider workplace diversity as a source of sustained competitive advantage, as it is not an easily imitable organizational characteristic. The presence of diverse individuals in important job positions can also offer visibility and legitimacy (Yang \& Konrad, 2011).

Studying attitudes to workplace diversity is extremely beneficial as diversity management policies and their desired effects rely on employees' attitudes (Lawrence, 1997). Researching attitudes of the employees toward diversity offers valuable information to decision-makers, giving them the baseline on diversity training requirements and the required diversity management policies (Traavik \& Adavikolanu 2016). Patrick and Kumar (2012) suggest that diversity attitudes may affect all organizational interactions. A positive attitude towards diverse team/group members can lead to increased cooperation, less sensitivity to intergroup bias, and increased motivation to achieve team objectives efficiently (van Knippenberg \& Schippers, 2007). Kundu and Mor (2017) propose that diversity attitudes of employees may even become an important predictor of organizational commitment and organizational performance. Diversity attitudes can also impact the team's ability to successfully implement diversity policies at an organizational level (Strauss et al., 2008). In this sense, results showing positive diversity attitudes have a high chance to be directly proportional to performance, motivation and loyalty.

Context plays an essential role in the diversity management discourse, as context drives the perception of diversity (Bacouel-Jentjens \& Yang, 2019). We choose to analyze the studies from different national contexts to have a base of comparison and observe how local context may influence employee's attitudes. Context is relevant when studying diversity as variables such as economic status, historical context, geographical location, traditions or religion interfere and influence the way individuals perceive different dimensions of diversity.

\section{OBJECTIVES AND RESEARCH METHODOLOGY}

The main objectives of this paper are:

1. identify the methods used by researches to measure diversity attitudes in public or private organizations;

2. determine the relation of context and attitudes to workplace diversity.

The current literature review was based on a systematic research of articles whose main topic comprised of workforce diversity and diversity management practices and included empirical research focused on measuring the attitudes of different types of employees regarding the existence of diversity in their professional environment.

In order to find the relevant studies, we searched specific keywords using the Web of Knowledge database. The key terms used included: "diversity management", "workplace diversity" or "workgroup diversity”, or "organizational diversity”, “diversity attitudes”, "employee attitudes toward diversity" or "perceived organizational diversity".

The first criterion applied when selecting relevant publication was the timeframe in which the articles were published, respectively the studies published in the last 12 years, starting from January 2008 until July 2020. The second criterion included the quality of the publication, namely the journal positioned inside the Web of Knowledge database. 
Notwithstanding, to avoid omitting relevant studies in the field of diversity management, we decided to include in the present paper a small number of non-ranked journals focused specifically on workforce diversity and perceived diversity inside organizations.

\section{RESULTS AND DISCUSSIONS}

\subsection{Studies by Methodology}

Attitudes of employees in relation to workplace diversity is a relatively new focus in the diversity management discourse. Hence, we considered it necessary to identify the methodologies preferred by researchers when measuring attitudes to workplace diversity, particularly aimed at employees.

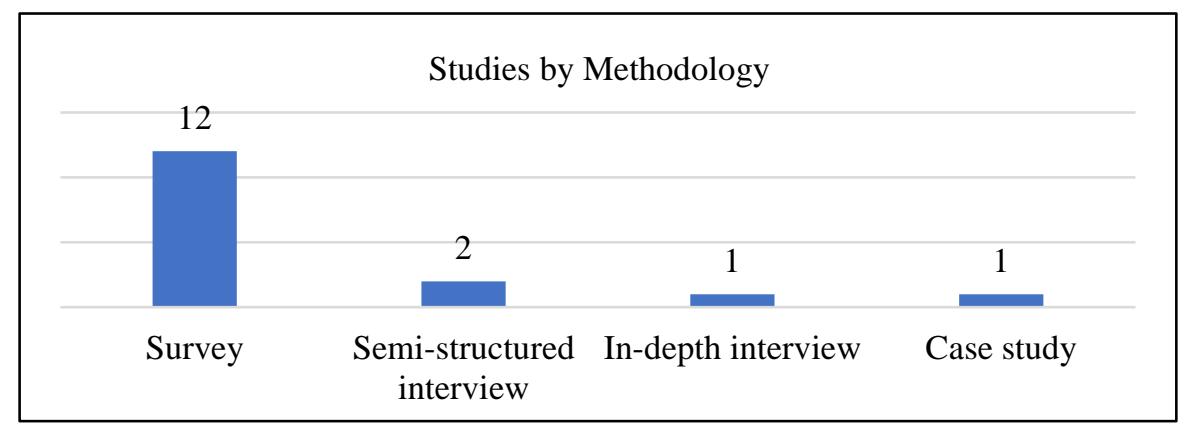

Figure 1. Studies by Methodology

Source: Own editing, based on the literature reviewed

In terms of the methodology of studies, as presented in Figure 1, 12 papers used surveys to measure attitudes toward workplace diversity, while in 3 articles, qualitative methods have been preferred, namely 2 semi-structured interviews and one in-depth interview. Lastly, only one article used a case study as the main research methodology. Arguing the methodology decision, researchers argued the reason why a quantitative measure was used. Scholars considered a questionnaire as the best option for the context of the study, in comparison with qualitative research because it is time-efficient, considering the high respondent number, it is also more focused and freer of interviewer bias (Davis et al., 2015; Hassi et al., 2015). In contrast, Cox (2004) argues for greater use of qualitative approaches in diversity research through the use of in-depth interviews and open-ended questioning when studying diversity. In the cases of all the quantitative studies, items or statements of the questionnaires were measured by using either 5-point, 6-point, or 9-point Likert scales.

Further, we have decided to pay close attention to the frameworks and literature that inspired the researchers to create the items and statements used as part of the surveys or interviews, as well as mention studies that created original survey statements.

One of the first instruments developed for measuring attitudes toward workplace diversity was created by De Meuse et al. (2007). The Workplace Diversity Survey (2001) contains 20 well though items that researchers can adapt and apply to diversity management studies in various contexts. Traavik and Adavikolanu (2016) have predicted the value of the previously mentioned survey and applied it in their research focused on diversity attitudes in India, Norway, and the Czech Republic. Hennekam and Tahssain-Gay (2015) conducted semi-structured interviews in The Netherlands and Morocco. The study followed Hofstede et al. (2010) cultural dimensions as the main framework. Hofstede's (2001) framework is widely used as it provides easy to use information involving online comparative data regarding all the countries cultural dimensions and relevant explanations related to the results.

Several scholars preferred to include in their studies more popular diversity scales or surveys as part of their studies. Walker et al. (2019) measured the attitudes toward diversity in the United States of 
America using the short form of the Miville-Guzman Universality-Diversity Scale (Miville et al., 1999). Attitudes towards diversity in Morocco were measured using a survey initially developed by Maricopa Community College from the USA (Hassi et al., 2015). One research from Australia, a developed country who is considered advanced in terms of diversity management polices inside the workplace included survey items inspired from editions of the national HR publications of the American Society for Human Resource Management and the Australian Human Resources Institute (Davis et al., 2015).

Two studies from the Indian IT sector had a different approach regarding the way respondents were classified and the survey conducted. Partick and Kumar (2012) decided to categorize the employees from the Indian IT sector as "diversity optimists," "diversity realists," or "diversity pessimists", creating an original survey based on the national context. In contrast, Kundu and Mor (2017) decided to select a survey adapted from the work of Soni (2000). Blouch and Azeem (2019) researched employee's diversity attitudes in the public and private health sector of Pakistan and the survey applied items adapted from Kundu and Mor (2017), the authors previously mentioned.

A selected number of statements will be provided next as we consider them to be valuable examples meant to inspire future researchers. All these items are part of the surveys or interviews of the selected papers and they are either original or based on previous literature research. Examples of positive statements from surveys include:

- "Diverse employees bring new perspectives to the organization” (Kundu \& Mor, 2017);

- "I feel at ease with people from backgrounds other than my own" and "I think that diverse viewpoints add value” (Kim et al., 2015);

- "Our company has clearly benefited from the workforce diversity it has" (Davis et al., 2015);

- "Diversity leads to harmony in organizations" (Traavik and Adavikolanu, 2016).

A statement example taken from a semi-structured interview is: "Have you experienced situations (positive or negative) at work when diversity issues were involved?” (Bacouel-Jentjens \& Yang, 2019).

\subsection{Diversity Dimensions}

In the diversity management discourse, there is an infinite number of diversity dimensions, however, the most researched diversity dimensions inside the business environment are age, ethnicity/nationality, gender, mental/physical ability, organizational role/function, race, religion, and sexual orientation (Köllen, 2019). Despite the continuous global changes, out of the dimensions presented above, the main focus of the diversity research still remains gender and race (Köllen, 2019; Ozturk \& Tatli, 2016). It is forecasted that in the future, age diversity could be the most researched dimension of the workforce diversity (Süß, 2008) and dimensions as sexual orientation and gender identity will also increase in relevance (Qin et al., 2014; Sabharwal et al., 2016).

Context, history, and location hold an important role when it comes to which diversity dimension has more relevance for a region or a country. An example unique to Eastern Asia shows that some of the most important diversity dimensions include: marital status of employees (Jaiswal \& Dyaram, 2018) or the residential status (Thomson et al., 2019), dimensions that are not considered to be of relevance in the Western society. Davis et al. (2015) indicate that diversity practices vary from country to country due to different contextual dimensions of each organization.

Related to Figure 2, we would like to note that a number of studies have emphasized more than one diversity dimension, those articles being counted multiple times. 


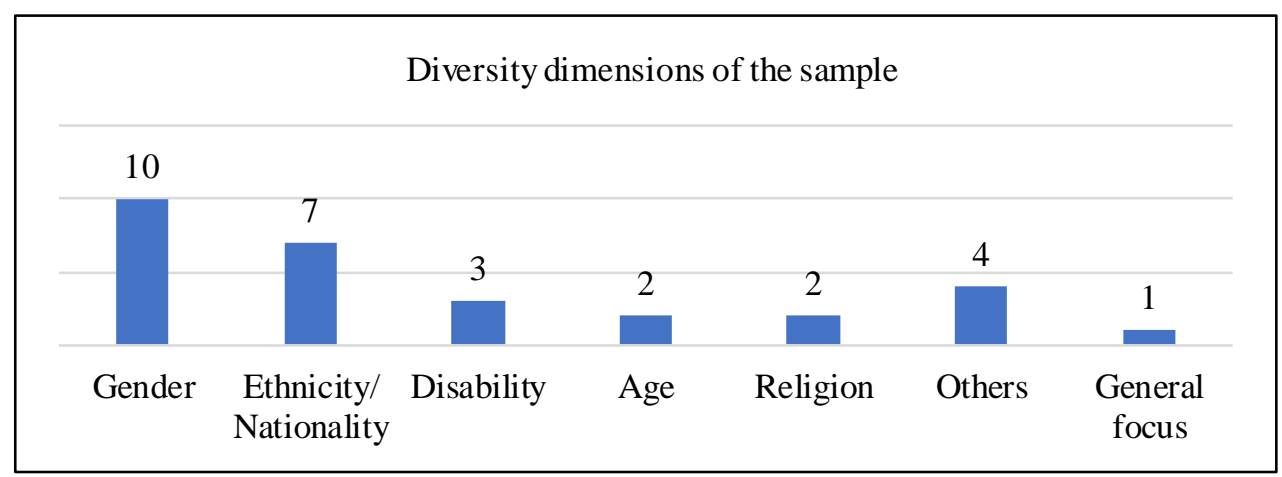

Figure 2. Diversity dimensions of the sample

Source: Own editing, based on the literature reviewed

In order to measure attitudes to workplace diversity, the authors used varied diversity dimensions to focus on in their methodologies. We believe that diversity dimensions are relevant to the context of the presented papers and their main findings. As seen in Figure 2, gender and ethnicity/nationality are the main diversity dimensions used to assess attitudes toward workplace diversity, followed by disability, age, religion. Other diversity dimensions influencing the context of the studies include education, caste, language, and culture. Authors from one study decided to follow a general diversity focus when measuring employee attitudes, not mentioning any diversity dimension throughout the research.

\subsection{How context influences attitudes to workplace diversity}

Context can be defined as "stimuli [...] that surround and thus exist in the environment external to the individual” (Mowday \& Sutton, 1993). For our research purpose, it is necessary to point out that different aspects related to the context have the power of positively or negatively interfere with diversity attitudes inside organizations (Johns, 2006).

Organizational approaches to diversity management are influenced by macro-national forces external to the workplace (Syed \& Murray, 2009). In this respect, workplace experiences of diverse employees are not only shaped by organizational and/or legal contexts but also by ethnic and religious factors (Hassi et al., 2015). Group cultures, organizational demography, national culture are also part of the contextual dimensions that may affect the attitudes of the workforce (BacouelJentjens \& Yang 2019).

Two studies were identified in the Indian IT sector, and results from both studies present a general neutral attitude toward workplace diversity, while the rest of employees display a positive attitude (Kundu \& Mor, 2017; Partick and Kumar, 2012). Employees from the financial industry in the United Arab Emirates also show indifferent attitudes toward diversity (Alserhan et al., 2010). Neutral attitude towards workplace diversity may arise in a context where diversity policies are born out of necessity rather than moral reasoning, combined with legal requirements (Alserhan et al., 2010; Partick and Kumar, 2012).

Taking into consideration the gender diversity dimension, similar to findings from different contexts, even if general attitudes toward diversity are considered positive, scholars measure specifically if there are differences with regard to how males and females perceive workplace diversity. The findings from the majority of studies suggest that men score lower in their attitudes compared with women employees (Blouch \& Azeem, 2019; Kim et al., 2015; Kundu \& Mor, 2017; Strauss et al., 2008; Traavik and Adavikolanu, 2016). The most significant difference between gender attitudes are the findings from Kim et al. (2015) based in the male-dominated South Korea. In this context, women perceive diversity significantly more positively than men. Other studies did not find any differences between gender and attitudes toward diversity inside organizations (Khan 
et al. 2010; Leca \& Vrânceanu, 2014). Results from none of the papers attest that male employees have a more positive attitude toward diversity in comparison with female employees.

The second most researched diversity dimension relates to ethnicity and/or nationality. Results of studies that focused on how ethnicity or nationality affects attitudes toward diversity at work present multiple inequalities. In the study of Hassi et al. (2015) foreign employees working in Morocco perceive diversity more positively than the Moroccan citizens working inside their country. Groeneveld (2011) propose that national minorities working inside a country will have a more positive attitude related to workplace diversity as the national employees because the diversity policies implemented in the organization will help them get equal status and similar opportunities as the local workers. Mor Barak et al. (1998) proposes a similar perspective presenting the fact that even though diversity management policies are meant to benefit everyone inside an organization, it is perceived differently by minority and majority groups. The authors suggest that minorities have the tendency to perceive diversity more positively as they are likely to consider themselves the beneficiaries of the diversity management policies inside organizations. Two studies from the United States of America and the United Kingdom focused on how race influences diversity attitudes. Walker et al. (2019) suggest that the attitude to diversity inside a group/team is positive when there is no dominant race/ ethnicity among team members. In contrast, racial minorities (nonwhites) were observed as having a more positive attitude related to diversity, in comparison with white employees (Strauss et al., 2008).

The influence of location on how employees perceive workplace diversity was clearly distinguished in the study of Khan et al. (2010). Location was a secondary variable in the research from the United Arab Emirates and findings reveal notable differences as employees from Dubai have a more positive attitude toward diversity compared with employees based in Abu-Dhabi (Khan et al., 2010). As Abu Dhabi city is considered more traditional than Dubai, respondents were less prone to accept and embrace diversity inside their organization.

Differences of attitudes are not only visible between nations, the results from one study based in France shows that employees may have a distinct personal attitude even in two different units of the same organization (Bacouel-Jentjens \& Yang, 2019). The authors suggest that the main context influencers, including workforce composition and social background of the employee have the strength to shape one's social identity, thus explaining why the attitudes toward diversity differ in the two researched working units. Schmid et al. (2012) also study the complexity of context and how it influences intergroup relations. The authors research the people living in ethnically diverse neighborhoods, main findings showing that being exposed to a diverse context will lead to a more complex identity structure, generating, in turn, less bias towards diverse individuals and a more positive attitude toward diversity and intergroup relations. Bacouel-Jentjens and Yang (2019) suggest that the powerful influence of context related to diversity perception may explain the inconsistent research results of diversity management and its organizational outcomes. Shifting to a smaller context, findings from a Romanian study investigating two different teams, part of the same department strongly suggests that the members of one team perceive diversity more positively compared with the other team (Leca \& Vrânceanu, 2014). As findings from the previously mentioned articles show, individuals' social identity was shaped in different contexts, thus explaining why some employees value diversity more and feel inspired and motivated by the existence of diversity in the workplace.

Industry and job category are other variables capable of influencing attitudes towards diversity. An example comes from the study of Hassi et al. (2015), where results indicate that attitudes to diversity of the employees working in the services industry are more positive in comparison with those working in agriculture or manufacturing. Related to the job category variable, findings from a study focused on the health sector in Pakistan indicate differences in how positively diversity was perceived based on hierarchal levels. The example shows that doctors, in comparison with 
paramedical and administrative staff, to have a more positive attitude related to diversity inside their organization (Blouch \& Azeem, 2019).

Job position can also influence the attitudes related to workplace diversity based on the findings from Davis et al. (2015). The research respondents included HR managers and non-HR managers in the Australian context and the main findings express a more positive attitude towards the workforce diversity of the HR employees in comparison with the non-HR employees on all measured items. The authors base this difference of attitudes mainly on the awareness and knowledge that HR employees hold on diversity-related topics, as they are the main initiators of diversity management policies.

It is valuable for decision-makers to study how attitudes related to diversity change over time and how culture influences these changes (Hennekam \& Tahssain-Gay, 2015). Two of the identified studies focused on applying Hofstede's (2001) cultural dimensions on a multi-national scale (Hennekam \& Tahssain-Gay, 2015; Traavik and Adavikolanu, 2016). The first cross-cultural study focused on observing how employee diversity attitudes differ in the same organization located in two countries, The Netherlands and Morocco and based on the cultural dimensions created by Hofstede et al. (2010) it was expected that the employees from The Netherlands to have a more positive attitude to diversity compared with those in Morocco (Hennekam \& Tahssain-Gay, 2015). Even as the hypothesis was confirmed, the scholars proved that cultural dimensions scores developed by Hofstede are rapidly changing, as findings from Morocco show an increasingly positive and open attitude toward diversity, while the results from The Netherlands present a decrease in diversity tolerance, especially considering the cultural diversity dimension. Traavik and Adavikolanu (2016) also confirmed that the cultural dimensions Hofstede (2001) and their influence related to diversity attitudes are changing. The study was conducted in India, Czech Republic, and Norway, and based on Hofstede's (2001) cultural dimensions, researchers expected Norway respondents to have the most positive attitudes toward workplace diversity in comparison with the other two countries. The results were not consistent with Hofstede as the respondents from the Czech Republic scored more positive for attitudes to workplace diversity than the Norwegians and Indians.

Considering context in the presented articles, in line with the main conclusions of Hennekam and Tahssain-Gay (2015), we may remark that besides cultural differences, there is a multitude of other variables as location, religion, traditions, economic development or education that play an important role in how employees perceive workplace diversity.

\section{CONCLUSION}

The main findings from the recent research concentrated on attitudes toward workplace diversity present limited generalizability of the results and high influence of context.

The present systematic review can motivate future scholars on measuring attitudes of workplace diversity taking into consideration the influence of context and choosing the methodology that is considered most suitable to help collect valuable data.

The preferred methodology of those researching the topic of attitudes related to organizational diversity was the survey, as it proved time efficient, considering the high sample sizes. Related to the diversity dimensions, gender and race are the most focused variables when measuring attitudes concerning diversity.

Findings show differences between the attitudes of employees related to workforce diversity in various contexts. There were recorded differences between countries, regions, industries, organizations, departments, teams, as well as between the attitudes of women and men, foreigners and nationals, and employees having different job positions. Different attitudes related to workforce diversity are expected by researchers, however, having access to this information is valuable for organizations and the individuals who have the task to create various diversity policies meant to 
improve performance and contribute to an inclusive environment. In a business environment, it is the duty of employers to measure attitudes to diversity and take corrective actions if necessary.

To have access to complete information from the researched environment we recommend scholars measuring attitudes toward diversity the use of mixed qualitative and quantitative methods and a general focus related to diversity dimensionality. In an organizational context, future studies should include in-depth interviews with the employees and employers in order to obtain detailed data concerning the perspectives of both the diversity policies initiators and the recipients.

\section{REFERENCES}

Alserhan, B. A., Forstenlechner, I. \& Al-Nakeeb, A. (2010). Employees' attitudes towards diversity in a non-western context. Employee Relations, 32(1), 42-55.

Bacouel-Jentjens, S. \& Yang, I. (2019). Do we see the same? Discrepant perception of diversity and diversity management within a company. Employee Relations, 41(3), 389-404. doi:10.1108/er-122017-0286.

Blouch, R. \& Azeem, M. F. (2019). Effects of perceived diversity on perceived organizational performance: Mediating role of perceived organizational justice. Employee Relations, 41(5), 10791097. doi: 10.1108/ER-05-2018-0150.

Chow, I. (2018). Cognitive diversity and creativity in teams: the mediating roles of team learning and inclusion. Chinese Management Studies, 12(2), 369-383.

Cox, T. (2004). Problems with research by organizational scholars on issues of race and ethnicity. The Journal of Applied Behavioral Science, 40(2), 124-145.

Davis, P. J., Frolova, Y. \& Callahan, W. (2015). Workplace diversity management in Australia: What do managers think and what are organizations doing? Equality, Diversity and Inclusion, 35(2), 8198.

De Meuse, K. P., Hostager, T. J. \& O’Neill, K. S. (2007). Longitudinal evaluation of senior managers' perceptions and attitudes of a workplace diversity training program. Human Resource Planning, 30(2), 38-46.

Groeneveld, S. (2011). Diversity and employee turnover in the Dutch public sector: Does diversity management make a difference? International Journal of Public Sector Management, 24(6), 594612.

Hassi, A., Foutouh, N. \& Ramid, S. (2015). Employee perception of diversity in Morocco: empirical insights. Journal of Global Responsibility, 6(1), 4-18. doi: 10.1108/JGR-01-2015-0001.

Hennekam, S. \& Tahssain-Gay, L. (2015). Changing attitudes towards diversity: The Netherlands and Morocco compared. Management Decision, 53(9), 2135-2155. doi: 10.18374/JIMS-17-1.2.

Hofstede, G. (2001). Culture's Consequences: Comparing Values, Behaviors, Institutions, and Organizations Across Nations, 2nd ed. Thousand Oaks: Sage Publications.

Hofstede, G., Hofstede, G. J. \& Minkov, M. (2010). Cultures and Organizations: Software of the Mind, 3rd ed. New York: McGraw-Hill.

Jaiswal, A. \& Dyaram, L. (2018). Diversity: a matter of reality or perception? International Journal of Organizational Analysis, 26(5), 798-811.

Johns, G. (2006). The essential impact of context on organizational behavior. Academy of Management Review, 31(2), 386-408.

Khan, T. M., Clear, F., Al-Kaabi, A. \& Pezeshki, V. (2010). An exploratory study of the effects of diversity dimensions and intervening variables on attitudes to diversity. Team Performance Management, 16(5/6), 289-308.

Kim, H. K., Lee, U. H. \& Kim, Y. H. (2015). The effect of workplace diversity management in a highly male-dominated culture. Career Development International, 20(3), 259-272.

Köllen, T. (2019). Diversity Management: A Critical Review and Agenda for the Future. Journal of Management Inquiry. doi:10.1177/1056492619868025. 
Kundu, S. C. \& Mor, A. (2017). Workforce diversity and organizational performance: a study of IT industry in India. Employee Relations, 39(2), 160-183. doi: 10.1108/ER-06-2015-0114.

Lawrence, B. S. (1997). Perspective - the black box of organizational demography. Organization Science, 8(1), 1-22.

Leca, I. \& Vranceanu, C. A. (2014). Multicultural teams: Moving from Diversity to Inclusion. CrossCultural Management Journal, 16(2), 361-368.

McGuire, D. \& Bagher, M. (2010). Diversity training in organizations: An introduction. Journal of European Industrial Training, 34(6), 493-505.

Mohr, A. \& Shoobridge, G. E. (2011). The role of multi-ethnic workforces in the internationalisation of SMEs. Journal of Small Business and Enterprise Development, 18(4), 748-763.

Mowday, R. T. \& Sutton, R. I. (1993). Organizational behavior: linking individuals and groups to organizational contexts. Annual Review of Psychology, 44, 195-229.

Ozturk, M. B. \& Tatli, A. (2016). Gender identity inclusion in the workplace: broadening diversity management research and practice through the case of transgender employees in the UK. The International Journal of Human Resource Management, 27(8), 781-802. doi: 10.1080/09585192.2015.1042902.

Patrick, H. A. \& Kumar, V. R. (2012). Managing Workplace Diversity: Issues and Challenges. SAGE Open, 2(2), 1-15. doi: 10.1177/2158244012444615.

Qin, J., Muenjohn, N. \& Chhetri, P. (2014). A review of diversity conceptualizations: Variety, trends, and a framework. Human Resource Development Review, 13(2), 133-157. doi: $10.1177 / 1534484313492329$.

Sabharwal, M., Levine, H. \& D’Agostino, M. (2016). A conceptual content analysis of 75 Years of diversity research in public administration. Review of Public Personnel Administration, 38(2), 248267. doi: $10.1177 / 0734371 X 16671368$.

Schmid, K., Hewstone, M. \& Al Ramiah, A. (2012). Neighborhood diversity and social identity complexity: implications for intergroup relations. Social Psychological and Personality Science, 4(2), 135-142.

Strauss, J. P., Sawyerr, O. \& Oke, A. (2008). Demographics, individual value structures, and diversity attitudes in the United Kingdom. Journal of Change Management, 8(2), 147-170. doi: 10.1080/14697010701799445.

Sü $\beta$, S. \& Kleiner, M. (2007). Diversity management in Germany: dissemination and design of the concept. The International Journal of Human Resource Management, 18(11), 1934-1953.

Syed, J. \& Murray, P. (2009). Multi-level challenges in diversity: Stories of Muslim migrant women in Australia. Human Resource Management Journal, 19(4), 413-432.

Thomson, S. B., Wei, W. X. \& Swallow, P. (2019). Equality and harmony: Diversity management in China. Chinese Management Studies, 13(1), 113-127.

Traavik, L. E. \& Adavikolanu, A. V. (2016). Attitudes towards diversity: Evidence from business school students from Norway, India and the Czech Republic. Cross Cultural and Strategic Management, 23(3), 450-466. doi: 10.1108/CCSM-12-2013-0189.

Van Knippenberg, D. \& Schippers, M. (2007). Work group diversity. Annual Review of Psychology, 58, 515-541.

Walker, S., Ruggs, E., Morgan, W. \& DeGrassi, S. (2019). Diverse perspectives on inclusion: Exploring the experiences of individuals in heterogeneous groups. Equality, Diversity and Inclusion: An International Journal, 38(1), 2-19.

Yang, Y. \& Konrad, A. M. (2011). Understanding Diversity Management Practices: Implications of Institutional Theory and Resource-Based Theory. Group and Organization Management, 36(1), 638. doi: 10.1177/1059601110390997.

Zapata-Barrero, R. \& Van Ewijk, A. R. (2011). Spheres of Diversities: From Concept to Policy. Barcelona: CIDOB. 